\title{
Pneumonic-type lung adenocarcinoma with different ranges exhibiting different clinical, imaging, and pathological characteristics
}

\author{
Ji-wen Huo ${ }^{1}$, Xing-tao Huang ${ }^{1}$, Xian Li ${ }^{2}$, Jun-wei Gong ${ }^{1}$, Tian-you Luo ${ }^{1}$ and Qi Li ${ }^{1 *}$
}

\begin{abstract}
Background: Pneumonic-type lung adenocarcinoma (PLADC) with different ranges might exhibit different imaging and clinicopathological features. This study divided PLADC into localized PLADC (L-PLADC) and diffuse PLADC (D-PLADC) based on imaging and aimed to clarify the differences in clinical, imaging, and pathologic characteristics between the two new subtypes.

Results: The data of 131 patients with L-PLADC and 117 patients with D-PLADC who were pathologically confirmed and underwent chest computed tomography (CT) at our institute from December 2014 to December 2020 were retrospectively collected. Patients with L-PLADC were predominantly female, non-smokers, and without respiratory symptoms and elevated white blood cell count and C-reactive protein level, whereas those with D-PLADC were predominantly male, smokers, and had respiratory symptoms and elevated white blood cell count and C-reactive protein level (all $p<0.05$ ). Pleural retraction was more common in L-PLADC, whereas interlobular fissure bulging, hypodense sign, air space, CT angiogram sign, coexisting nodules, pleural effusion, and lymphadenopathy were more frequent in D-PLADC (all $p$ <0.001). Among the 129 patients with surgically resected PLADC, the most common histological subtype of L-PLADC was acinar-predominant growth pattern $(76.7 \%, 79 / 103)$, whereas that of D-PLADC was invasive mucinous adenocarcinoma $(80.8 \%, 21 / 26)$. Among the 136 patients with EGFR mutation status, L-PLADC had a significantly higher EGFR mutation rate than D-PLADC $(p<0.001)$.
\end{abstract}

Conclusions: L-PLADC and D-PLADC have different clinical, imaging, and pathological characteristics. This new imaging-based classification may help improve our understanding of PLADC and develop personalized treatment plans, with concomitant implications for patient outcomes.

Keywords: Pneumonic-type lung adenocarcinoma, Tomography (X-ray computed), Epidermal growth factor receptor

*Correspondence: lq781222711@163.com

${ }^{\dagger} \mathrm{JH}$ and $\mathrm{XH}$ contributed equally to this work and share the first authorship.

${ }^{1}$ Department of Radiology, The First Affiliated Hospital of Chongqing Medical University, No. 1 Youyi Road, Yu zhong District, Chongqing 400016, China

Full list of author information is available at the end of the article

\section{Key points}

- L-PLADC and D-PLADC have different clinical and imaging findings.

- Acinar-predominant growth pattern is the most common histological subtype of L-PLADC.

- Invasive mucinous adenocarcinoma is the most common histological subtype of D-PLADC. 
- L-PLADC has a significantly higher EGFR-mutations rate than that of D-PLADC.

\section{Background}

Lung adenocarcinoma (LADC) is the most common histological type of lung cancer [1]. It has a wide variety of manifestations on computed tomography (CT), and sometimes can represent nonobstructive focal or diffuse pulmonary consolidation, which is usually misdiagnosed as pneumonia [2]. This type of LADC is also known as pneumonic-type LADC (PLADC) [3, 4]. Previous studies demonstrated that PLADC corresponds to bronchioloalveolar carcinoma (BAC) with a predominant mucinous histologic subtype, and several investigators have studied the imaging features of PLADC [3-7]. Jung et al. [4] noted that $\mathrm{CT}$ findings favored the diagnosis of PLADC based an irregular air bronchogram and a bulging fissure. Aquino et al. [5] reported that CT findings suggestive of PLADC included unresolving peripheral consolidation and coexisting nodules, whereas no significant differences were observed in air bronchogram, leafless tree, bulging fissure, cysts or cavities, low-attenuation consolidation, and CT angiogram sign between pneumonic-type $\mathrm{BAC}$ and infectious pneumonia. Im et al. [6] found that CT findings of pneumonic-type BAC included low-attenuation consolidation and CT angiogram sign. Obviously, some of these findings are inconsistent, which may lead to delayed diagnosis and unnecessary biopsies. Moreover, the term consolidation seems to be confusing as the definition of involved range differs among institutions [4-8]. Previous observations of our group in clinical practice showed that PLADC with different ranges might exhibit different imaging and clinicopathological features. Therefore, we speculated that the different outcomes reported in the literature among CT findings of PLADC are owing to the fact that PLADC is a heterogeneous group in which different patterns might be identified based on clinical, radiological, and histopathological findings.

To date, little research has focused on the characteristics of PLADC involving focal or diffuse area of the lung. In this study, we classified PLADC into localized PLADC (L-PLADC) and diffuse PLADC (D-PLADC) based on imaging and aimed to investigate whether clinical, imaging, and pathological characteristics of L-PLADC were different from those of D-PLADC, and if so, we intended to assess these differences.

\section{Materials and methods Patients}

The study protocol was approved by the ethics committee of our institute, and the need for informed consent was waived owing to the retrospective nature of the study. A total of 7248 patients with LADC from December 2014 to December 2020 were initially included. Inclusion criteria were as follows: (1) patients who underwent chest CT scan at our institute and (2) those confirmed pathologically by surgical resection, biopsy, and cytology. Subsequently, two experienced radiologists with $>10$ years of experience in chest imaging interpreted $\mathrm{CT}$ images on a picture archiving and communication system (PACS) workstation (Vue PACS, Carestream) together and classified these patients into three categories: L-PLADC, D-PLADC, and non-PLADC. L-PLADC was defined as localized consolidation involving $<50 \%$ of the area of a lobe. D-PLADC was characterized as diffuse consolidation involving $\geq 50 \%$ of the area of a lobe or lobes. NonPLADC refers to tumors that could not be classified as L-PLADC or D-PLADC. Among them, 165 patients were with L-PLADC, 143 with D-PLADC, and 6940 with non-PLADC. Finally, patients with PLADC were further screened according to the following exclusion criteria: (1) patients with a history of chemotherapy, radiotherapy, or other oncologic therapy before CT scans and (2) with an interval of $>1$ month between $\mathrm{CT}$ imaging and subsequent pathological analysis. In addition, clinical data of all patients, including age, sex, smoking history, respiratory symptoms, laboratory results, diagnostic methods, and clinicopathologic TNM stages at the time of diagnosis, were collected.

\section{Computed tomography protocols}

Chest CT examinations were performed using the Discovery CT750HD (GE Healthcare), Light speed VCT (GE Healthcare), or Somatom Definition Flash (Siemens Healthcare) scanner. All patients underwent CT scanning in the supine position at the end of inspiration during a single breath hold. Imaging parameters were as follows: tube voltage, $120 \mathrm{kVp}$; tube current, $100-250 \mathrm{~mA}$; and scanning slice thickness/interval, $5 \mathrm{~mm} / 5 \mathrm{~mm}$. A total of 170 patients $(170 / 248,68.5 \%)$ received contrastenhanced scanning and were injected with nonionic iodinated contrast medium (iohexol $300 \mathrm{mg}$ iodine $/ \mathrm{mL}$; Omnipaque, GE Healthcare) at a dosage of $1.5 \mathrm{~mL} / \mathrm{kg}$ of body weight (total volume: $80-110 \mathrm{~mL}$ ) using a dualhigh-pressure injector via the antecubital vein at a flow rate of $3.0 \mathrm{~mL} / \mathrm{s}$. This was followed by injection of $50-\mathrm{mL}$ saline solution. Acquisition times in arterial and delayed phases were triggered at 30 and $120 \mathrm{~s}$, respectively, after the start of contrast medium injection. All images were reconstructed with a section thickness and slice interval of $0.625 \mathrm{~mm}$ or $1 \mathrm{~mm}$ and $0.625 \mathrm{~mm}$ or $1 \mathrm{~mm}$, respectively, for analysis. 


\section{Computed tomography image analysis}

The aforementioned radiologists analyzed the CT features of tumors on our PACS workstation, and any disagreements were resolved by discussion until a consensus was reached. The following data were carefully observed and recorded: (1) distribution (single lobe [left upper and lower lobes; right upper, middle, and lower lobes], unilateral multiple lobes, and bilateral multiple lobes); (2) presence of interlobular fissure bulging, hypodense sign (low-density area within consolidation without enhancement), air space (air attenuation in lesions), irregular air bronchogram (air-filled bronchus manifesting as dilatation, rigidity, or narrowing), CT angiogram sign (branching pulmonary vessels extending $>3 \mathrm{~cm}$ along a single channel), ground-glass opacity (GGO, increased attenuation without obscuration of underlying lung vessels), coexisting nodules, pleural retraction (one or more linear or cord-like structures connected between the tumor and pleura in the lung window images), pleural effusion, and lymphadenopathy (hilar or mediastinal lymph nodes with a short-axis diameter of $>1 \mathrm{~cm}$ ). Clinical cancer stages of LADC were evaluated according to the 8th edition of the American Joint Committee on cancer staging system for non-small-cell lung cancer [9].

\section{Histochemical examination}

All selected specimens of PLADC stained with hematoxylin and eosin were analyzed by a pathologist with 12 years of experience in pathological diagnosis of the lung. LADC was determined according to the LADC classification system issued in 2011 [10]. The percentage of each growth pattern (acinar, solid, papillary, micropapillary, lepidic, etc.) in the tumor was semiquantitatively measured in 5\% increments, and that with the largest percentage was considered a predominant pattern. Accordingly, the histologic subtype of invasive LADC was described based on its predominant pattern.

\section{Epidermal growth factor receptor mutation analysis}

Surgical specimens, biopsy specimens, and hydrothorax fallen cells of tumors were used for molecular analysis. The epidermal growth factor receptor (EGFR) mutation status (exons 18-21) was examined using a real-time polymerase chain reaction-based amplification refractory mutation system using the Human EGFR Gene Mutations Detection Kit (AmoyDx, Xiamen, China).

\section{Statistical analysis}

Statistical analyses were performed using the International Business Machines Statistical Package for the Social Sciences Statistics for Windows (version 25.0; IBM Corp., Armonk, NY, USA). Single-sample
Kolmogorov-Smirnov analysis was used to test the variance in measurement homogeneity. Normally distributed quantitative data are expressed as mean \pm standard deviation, and non-normally distributed data are presented as median \pm interquartile range, whereas categorical variables were expressed as numbers and percentages. The Chi-square test was employed to compare frequencies of different clinical factors, CT features, and EGFR mutation status between L-PLADC and D-PLADC. A two-tailed $p$ value of $<0.05$ was considered as statistically significant.

\section{Results \\ Study population}

In total, 308 patients with PLADC were initially selected from 7248 patients who were pathologically confirmed with LADC. Among them, 60 patients were excluded owing to an antitumor therapy history before chest CT scan $(n=12)$ and interval of $>1$ month between CT imaging and subsequent pathological analysis $(n=48)$. Finally, 131 patients with L-PLADC and 117 patients with D-PLADC were enrolled for analysis. The flow diagram for this study is shown in Fig. 1. Among the 131 patients with L-PLADC, 108 (82.4\%) were confirmed by surgical resection, 14 (10.7\%) by bronchoscopy biopsy, six (4.6\%) by transthoracic needle biopsy, and three $(2.3 \%)$ by cytology; 100 patients (76.3\%) were in stages I-II and $31(23.7 \%)$ in stages III-IV. Among the 117 patients with D-PLADC, nine (7.7\%) were confirmed by surgical resection, $44(37.6 \%)$ by bronchoscopy biopsy, 23 (19.7\%) by transthoracic needle biopsy, and 41 (35.0\%) by cytology; seven patients (6.0\%) were in stages I-II and 110 (94.0\%) in stages III-IV.

\section{Comparison of clinical characteristics between L-PLADC and D-PLADC}

Clinical data of patients with L-PLADC and D-PLADC are given in Table 1 . The average age of patients with L-PLADC was 62 years (range 35-62 years) and that of patients with D-PLADC was 61 years (range 23-84 years). No significant difference was observed in the age between both groups $(p>0.05)$. C-reactive protein level was assessed in 116 patients who underwent this test. The number of patients in the D-PLADC group who had respiratory symptoms, including cough and sputum, as well as elevated white blood cell count and C-reactive protein level was greater than that of those in the L-PLADC group (all $p<0.05$ ). However, no significant differences were observed in symptoms such as fever, blood in sputum, hemoptysis, and chest pain between both groups (all $p>0.05$ ). 


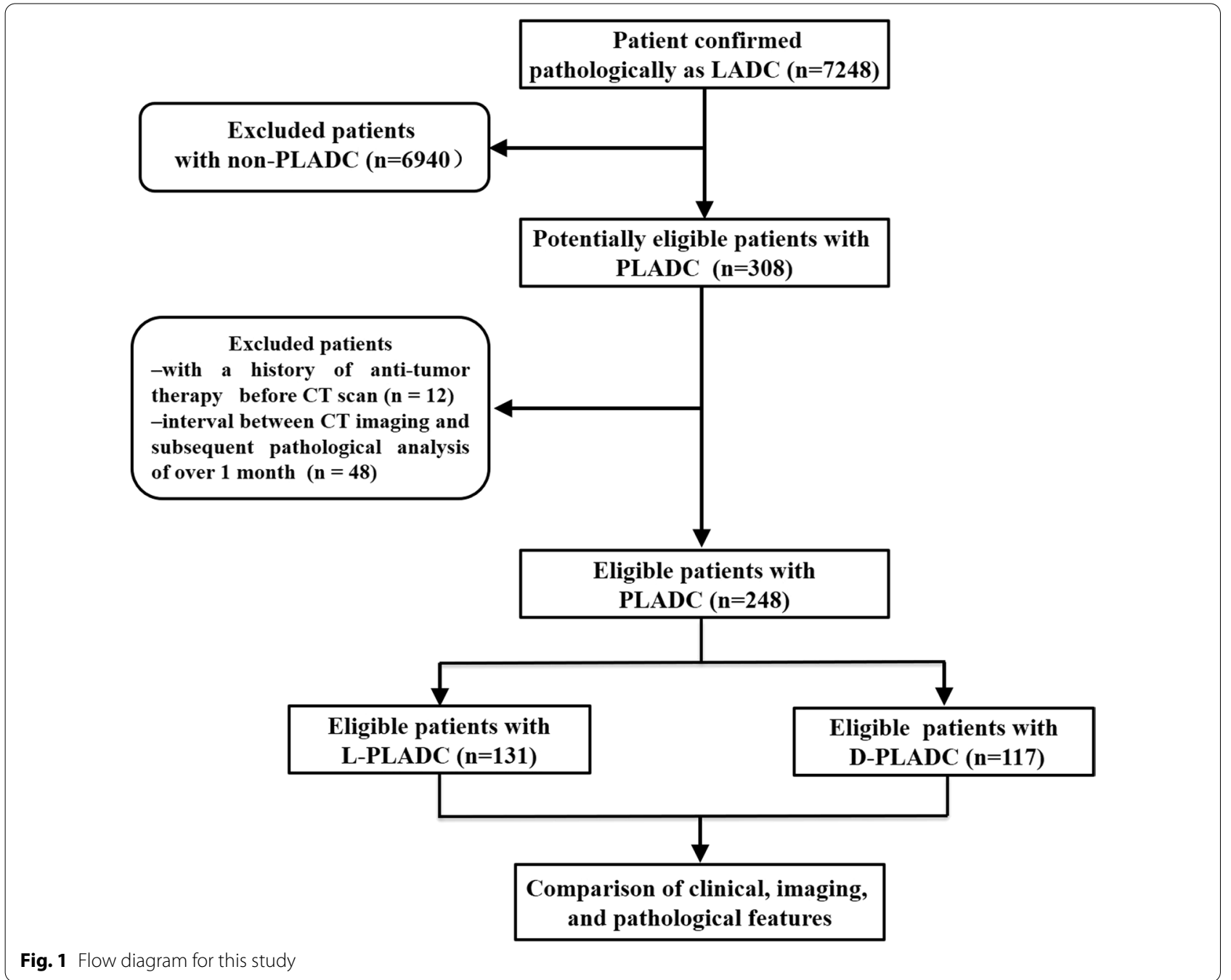

\section{Comparison of $C T$ findings between L-PLADC and D-PLADC}

CT features of patients with L-PLADC and D-PLADC are given in Table 2. Hypodense and CT angiogram signs were assessed in 170 patients who underwent contrast-enhanced scanning. Bilateral multiple lobes distribution was most common in the D-PLADC group. Interlobular fissure bulging, hypodense sign, air space, CT angiogram sign, coexisting nodules, pleural effusion, and lymphadenopathy were more common in patients with D-PLADC, whereas pleural retraction was more frequently seen in patients with L-PLADC (all $p<0.001$ ). Both D-PLADC and L-PLADC showed high percentages of irregular air bronchogram and GGO. However, no significant differences were observed in irregular air bronchogram and GGO between both groups (all $p>0.05$; Figs. 2A, 3A-E).

\section{Comparison of histological subtypes between L-PLADC and D-PLADC}

Histological subtypes of patients with L-PLADC and D-PLADC are shown in Fig. 4. In total, 103 patients with L-PLADC and 26 patients with D-PLADC were confirmed as invasive LADC with identifiable growth patterns. Acinar-predominant growth pattern was found to be the most common histological subtype for patients with L-PLADC $(76.7 \%, 79 / 103)$, followed by papillarypredominant growth pattern $(9.7 \%, 10 / 103)$ and lepidicpredominant growth pattern $(4.9 \%, 5 / 103)$ (Figs. 2C, 4A). Invasive mucinous adenocarcinoma was the most common subtype in patients with D-PLADC $(80.8 \%, 21 / 26)$, followed by acinar-predominant growth pattern $(11.5 \%$, $3 / 26)$ and papillary-predominant growth pattern $(7.7 \%$, 2/26) (Figs. 3F, 4B). 
Table 1 Comparison of clinical characteristics between L-PLADC and D-PLADC

\begin{tabular}{|c|c|c|c|}
\hline Characteristic & Patients with L-PLADC $(n=131)$ & Patients with D-PLADC $(n=117)$ & $p$ value \\
\hline \multicolumn{4}{|l|}{ Age (years) } \\
\hline Means \pm standard deviations & $62 \pm 9$ & $61 \pm 11$ & \multirow[t]{2}{*}{$0.404^{\mathrm{a}}$} \\
\hline Range & $35-62$ & $23-84$ & \\
\hline \multicolumn{4}{|l|}{ Sex } \\
\hline Male & $43 / 131(32.8 \%)$ & $72 / 117(61.5 \%)$ & \multirow[t]{2}{*}{$<0.001$} \\
\hline Female & $88 / 131(67.2 \%)$ & $45 / 117(38.5 \%)$ & \\
\hline \multicolumn{4}{|l|}{ Smoking } \\
\hline Non-smokers & 97/131 (74.0\%) & $52 / 117(44.4 \%)$ & \multirow[t]{2}{*}{$<0.001^{b}$} \\
\hline Smokers & $34 / 131(26.0 \%)$ & $65 / 117(55.6 \%)$ & \\
\hline \multicolumn{4}{|l|}{ Respiratory symptoms } \\
\hline Fever & $7 / 131(5.3 \%)$ & $9 / 117(7.7 \%)$ & $0.452^{b}$ \\
\hline Cough & $54 / 131(41.2 \%)$ & 103/117 (88.0\%) & $<0.001^{b}$ \\
\hline Sputum & 42/131 (32.1\%) & $77 / 117(65.8 \%)$ & $<0.001^{b}$ \\
\hline Blood in sputum & 13/131 (9.9\%) & $13 / 117(11.1 \%)$ & $0.761^{b}$ \\
\hline Hemoptysis & $8 / 131(6.1 \%)$ & $11 / 117(9.4 \%)$ & $0.330^{b}$ \\
\hline Chest pain & $16 / 131(12.2 \%)$ & $13 / 117(11.1 \%)$ & $0.787^{b}$ \\
\hline No symptoms & $62 / 131(47.3 \%)$ & $5 / 117(4.3 \%)$ & $<0.001^{b}$ \\
\hline \multicolumn{4}{|l|}{ Laboratory results } \\
\hline Elevation of white blood cell count & $13 / 131(9.9 \%)$ & $41 / 117(35.0 \%)$ & $0.003^{b}$ \\
\hline Elevation of C-reactive protein level ${ }^{\mathrm{c}}$ & 10/32 (31.3\%) & 66/84 (78.6\%) & $<0.001^{b}$ \\
\hline
\end{tabular}

\section{EGFR mutation analysis}

A total of 136 patients with PLADC underwent molecular analyses of EGFR mutation. Among them, 64 patients had L-PLADC and 76.6\% (49/64) were positive for EGFR mutations, including 22 patients harboring a single exon 19 deletion mutation, 21 harboring a single L858R mutation, and six harboring other mutation subtypes; 72 patients had D-PLADC and 38.9\% (28/72) were positive for EGFR mutations, including 18 patients harboring a single 19 exon deletion mutation, four harboring a single L858R mutation, and six harboring other mutation subtypes. EGFR mutation rate of L-PLADC was significantly higher than that of D-PLADC $(p<0.001)$.

\section{Discussion}

This study aimed to investigate whether it is reasonable to divide PLADC into two new subtypes according to different ranges of lung involvement. To our knowledge, this is the first study to focus on differences in clinical, imaging, and pathological features between D-PLADC and L-PLADC. Several major findings were noted in this study.

First, we found that patients with L-PLADC were predominantly women and non-smokers, whereas those with D-PLADC were predominantly men and smokers. Studies have indicated that a high proportion of patients with LADC are women and non-smokers [11, 12], which is consistent with the demographic characteristics of patients with L-PLADC, but contradicts with those of patients with D-PLADC. Considering that L-PLADC and D-PLADC are new imaging-based subtypes, few studies have focused on their differential clinical features. Future studies with larger sample sizes are warranted to substantiate our findings. Furthermore, our results showed that the number of patients with L-PLADC without respiratory symptoms and elevated white blood cell count and C-reactive protein level was higher than those with D-PLADC without these symptoms. Chu et al. [13] indicated that the number of patients with lung cancers who were asymptomatic was higher than that of those with inflammatory lesions. Yao et al. [14] have showed that the C-reactive protein is a sensitive and nonspecific inflammatory marker, and its increased level is highly indicative of infection. Hence, these clinical characteristics of L-PLADC are similar to those reported in previous studies about lung cancer; however, some features of D-PLADC are contrary to those reported in the literature and often overlap with pulmonary infection, 
Table 2 Comparison of CT features between L-PLADC and D-PLADC

\begin{tabular}{|c|c|c|c|}
\hline CT Features & $\begin{array}{l}\text { Patients with } \\
\text { L-PLADC } \\
(n=131)\end{array}$ & $\begin{array}{l}\text { Patients with } \\
\text { D-PLADC } \\
(n=117)\end{array}$ & $p$ value $^{\mathrm{a}}$ \\
\hline \multicolumn{4}{|l|}{ Distribution } \\
\hline Single lobe & 131/131 (100\%) & $36 / 117(21.1 \%)$ & - \\
\hline Right upper lobe & 49/131 (37.4\%) & $6 / 117(5.1 \%)$ & - \\
\hline Right middle lobe & 12/131 (9.2\%) & $0 / 117(0 \%)$ & - \\
\hline Right lower lobe & 22/131 (16.8\%) & 14/117 (12.0\%) & - \\
\hline Left upper lobe & $35 / 131(26.7 \%)$ & $6 / 117(5.1 \%)$ & - \\
\hline Left lower lobe & 13/131 (9.9\%) & 10/117 (8.5\%) & - \\
\hline Right multiple lobes & 0/131 (0\%) & 20/117 (17.1\%) & \\
\hline Left multiple lobes & 0/131 (0\%) & $5 / 117(4.3 \%)$ & - \\
\hline $\begin{array}{l}\text { Bilateral multiple } \\
\text { lobes }\end{array}$ & 0/131 (0\%) & $56 / 117(47.9 \%)$ & - \\
\hline $\begin{array}{l}\text { Interlobular fissure } \\
\text { bulging }\end{array}$ & 8/131 (6.1\%) & 48/117 (41.0\%) & $<0.001^{a}$ \\
\hline Hypodense sign ${ }^{\text {b }}$ & $22 / 82(26.8 \%)$ & 79/88 (89.8\%) & $<0.001^{a}$ \\
\hline Air space & 40/131 (30.5\%) & 68/117 (58.1\%) & $<0.001^{\mathrm{a}}$ \\
\hline $\begin{array}{l}\text { Irregular air broncho- } \\
\text { gram }\end{array}$ & $90 / 131(68.7 \%)$ & $81 / 117$ (69.2\%) & $0.928^{a}$ \\
\hline $\mathrm{CT}$ angiogram sign ${ }^{\mathrm{b}}$ & $22 / 82(21.8 \%)$ & 79 /88 (89.8\%) & $<0.001^{\mathrm{b}}$ \\
\hline GGO & 101/131 (77.1\%) & $94 / 117(80.3 \%)$ & $0.534^{\mathrm{a}}$ \\
\hline Coexisting nodules & 15/131 (11.5\%) & $73 / 117$ (62.4\%) & $<0.001^{\mathrm{a}}$ \\
\hline Pleural retraction & 102/131 (77.9\%) & $8 / 117(6.8 \%)$ & $<0.001^{a}$ \\
\hline Pleural effusion & 6/131 (4.6\%) & $72 / 117(61.5 \%)$ & $<0.001^{\mathrm{a}}$ \\
\hline Lymphadenopathy & 13/131 (9.9\%) & $53 / 117(45.3 \%)$ & $<0.001^{\mathrm{a}}$ \\
\hline
\end{tabular}

$\mathrm{CT}$, computed tomography; L-PLADC, localized pneumonic-type lung adenocarcinoma; D-PLADC, diffuse pneumonic-type lung adenocarcinoma; GGO, ground-glass opacity

${ }^{\text {a }}$ Chi-squared test

${ }^{\mathrm{b}}$ Hypodense sign and $\mathrm{CT}$ angiogram sign was not assessed in 78 patients who did not undergo contrast-enhanced scanning

which may result in a diagnostic error [13, 14]. Familiarity with these special clinical features of D-PLADC may contribute to its early diagnosis. We speculated that involvement of more lobes leads to a higher frequency of respiratory symptoms in patients with D-PLADC. Our results showed that a higher number of patients with D-PLADC were in stages III-IV and had pleural effusion and lymphadenopathy, which were suggestive of a poor prognosis. Previous studies have demonstrated that an inflammatory reaction is closely related to the development and progression of malignant tumors, and increase in some inflammatory indexes such as C-reactive protein and neutrophils is associated with poor prognosis of tumors $[15,16]$. This may be a good explanation for the findings of the present study.

Next, we found that imaging features were diverse between L-PLADC and D-PLADC. The present study showed that pleural retraction was closely related to L-PLADC, whereas interlobular fissure bulging, hypodense sign, air space, CT angiogram sign, and coexisting nodules were closely correlated with D-PLADC. Possible causes underlying these findings could likely be attributed to the histological subtype differences between L-PLADC and D-PLADC. According to our results, L-PLADC showed a high frequency of acinar-predominant subtype, whereas D-PLADC had a high occurrence of invasive mucinous adenocarcinoma. Pleural retraction is indicative of tumor contraction resulted from the narrowing or collapse of alveolar spaces or from fibrotic areas in tumors, which is generally observed in patients with non-mucinous adenocarcinoma [17]. Generally, invasive mucinous adenocarcinoma contains columnar or goblet cells with abundant intracellular or extracellular mucus admixed with invasive adenocarcinoma patterns with stromal invasion. Tumor cells usually grow along the alveolar wall and secrete abundant mucin, thereby filling the alveolar lumina, which may cause gelatinous parenchymal consolidation and increased volume of the involved lung parenchyma $[3,18]$, contributing to interlobular fissure bulging and hypodense sign. After intravenous injection of contrast medium, the consolidated lung typically manifests as an area of low attenuation on $\mathrm{CT}$, allowing an enhanced branching of pulmonary vessels to be identified as CT angiogram sign. The presence of air space may be false in that tumor cell invades the bronchus and forms a unidirectional check-valve, which may allow air in but not out, resulting in the formation of a pseudo-cavity $[3,7,19,20]$. The coexisting nodules may be associated with multicentric growth or metastasis of tumors [21]. Consistent with our findings, some investigators showed that interlobular fissure bulging, low attenuation within consolidation, air space, CT angiogram sign, and coexisting nodules are strongly suggestive of PLADC [3-8, 17-20]. Our results also indicated that irregular air bronchogram and GGO were frequently detected in both subtypes of PLADC, which conforms to the results of some investigators $[4,7,22]$. Irregular air bronchogram may be associated with tumor invasion or desmoplastic reaction [22], whereas GGO may correspond to incomplete filling of the alveolar spaces and slight thickening of the alveolar wall owing to infiltration or mucus secretion by tumor cells [23]. Additionally, pleural effusion and lymphadenopathy were found to be more common in D-PLADC than L-PLADC, indicating a poor prognosis in patients with D-PLADC; this result is consistent with the results of other scholars, who stated that the pneumonic type of lung invasive mucinous adenocarcinoma correlated with poor outcome [24-26]. Therefore, this new imaging-based typing of PLADC may be helpful for us to better understand its CT findings and predict its prognosis. 

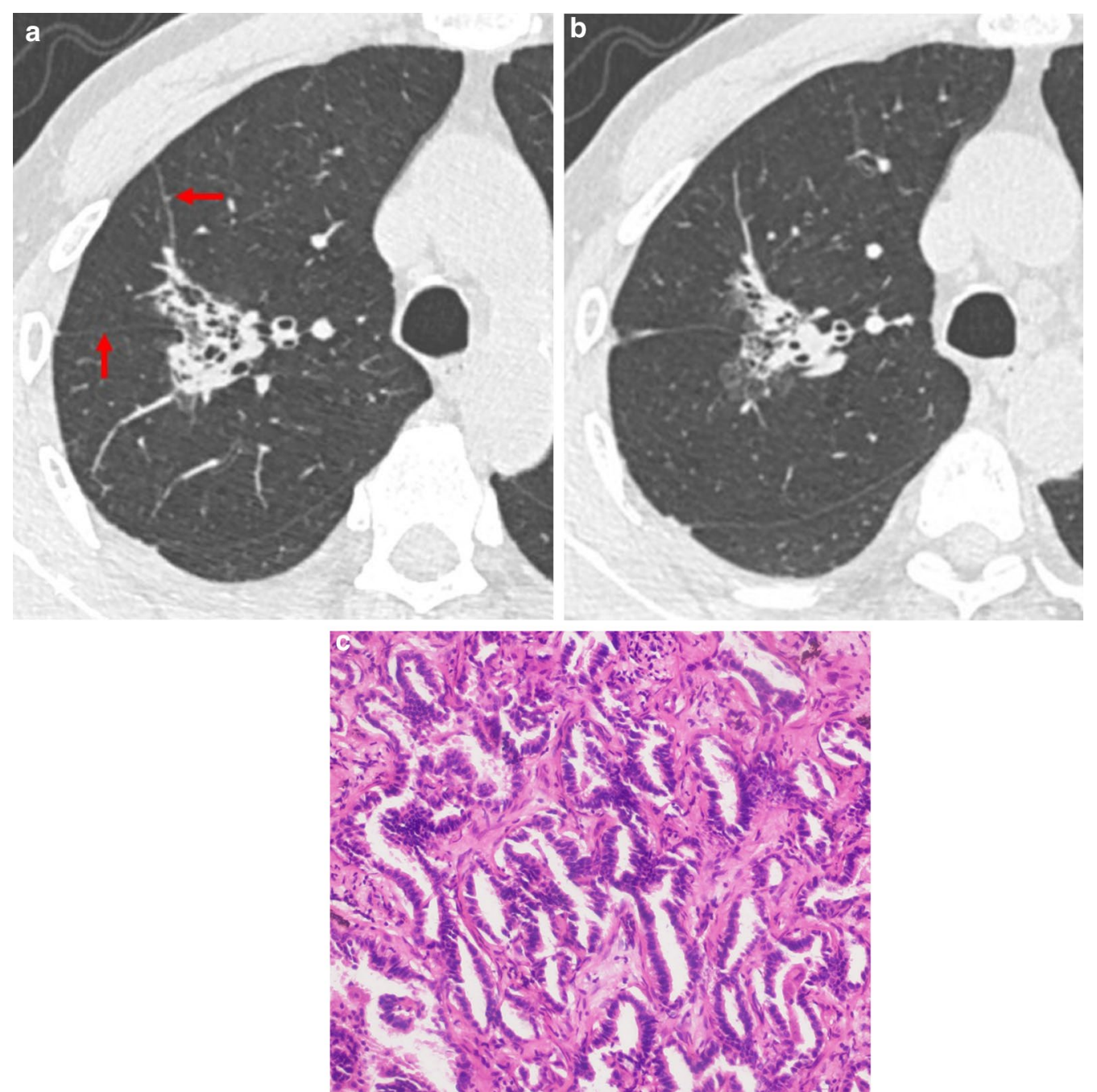

Fig. 2 L-PLADC in a 65-year-old man without symptoms. a, b Axial CT images of the lung window indicate a localized consolidation with irregular air bronchogram, GGO component, and pleural retraction (red arrow) in the right upper lobe. c Photomicrograph (hematoxylin and eosin staining, $\times 200$ ) of surgical specimens confirmed LADC with an acinar-predominant pattern

Furthermore, our findings showed that PLADC with focal and diffuse consolidation had different histological subtypes and EGFR mutation status. Our results indicated that L-PLADC showed a strong tendency for acinar-predominant subtype and EGFR positive mutations, whereas D-PLADC was closely related to invasive mucinous adenocarcinoma and EGFR negative mutations. Liu et al. [27] evaluated the clinical-radiologicalpathological characteristics and prognosis of advanced pneumonic-type lung cancer, which predominantly involved diffuse area of lung, and found that the majority of patients had invasive mucinous adenocarcinoma and correlated with the absence of EGFR mutations. Liu et al. [8] investigated the CT features that correlated with EGFR mutations in surgically resected pneumonic-type lung cancer, which predominantly involved the focal area of lung, and found that most patients were with acinarpredominant invasive adenocarcinoma and correlated with the presence of EGFR mutations. Our results were in agreement with previous results. Some scholars demonstrated that EGFR mutations correlated with women and non-smokers $[28,29]$. A prior study conducted by Yanagawa et al. [30] revealed that EGFR mutations had high frequency among some histological subtypes of adenocarcinoma, including adenocarcinoma in situ (62\%), minimally invasive adenocarcinoma (60\%), lepidic (77\%), papillary (50\%), acinar (49\%), and micropapillary (43\%), but a relatively low frequency in solid (28\%) and invasive mucinous adenocarcinoma (0\%). Therefore, the relatively high expression of EGFR mutations in patients with 

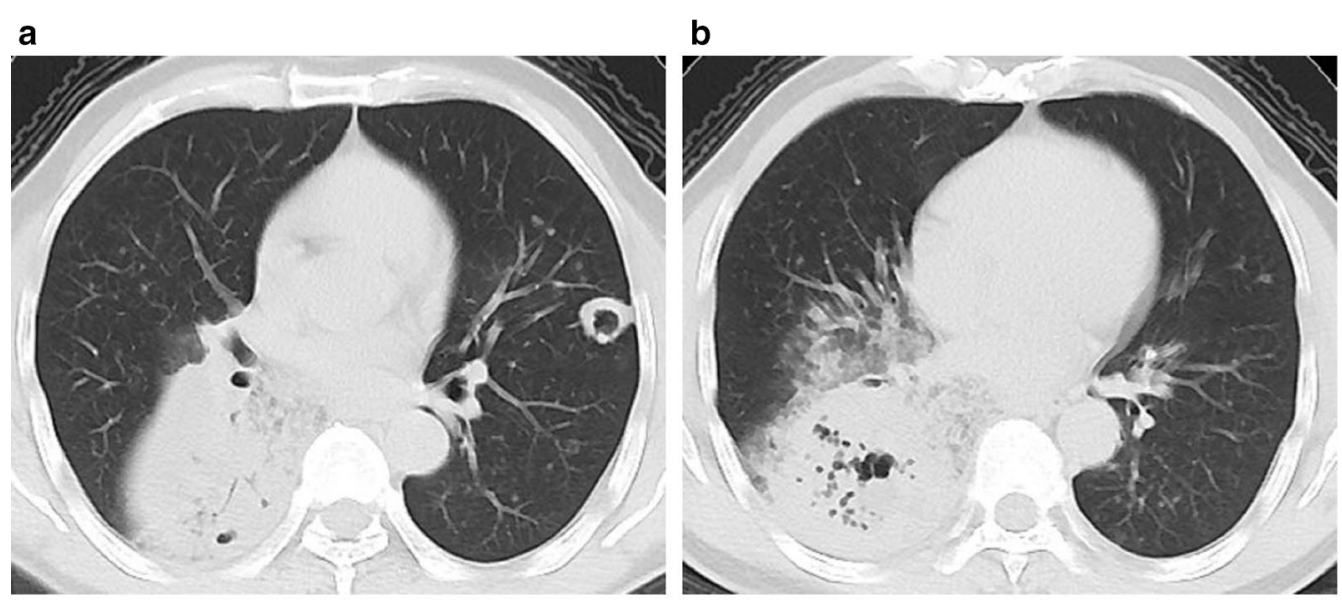

\section{C}

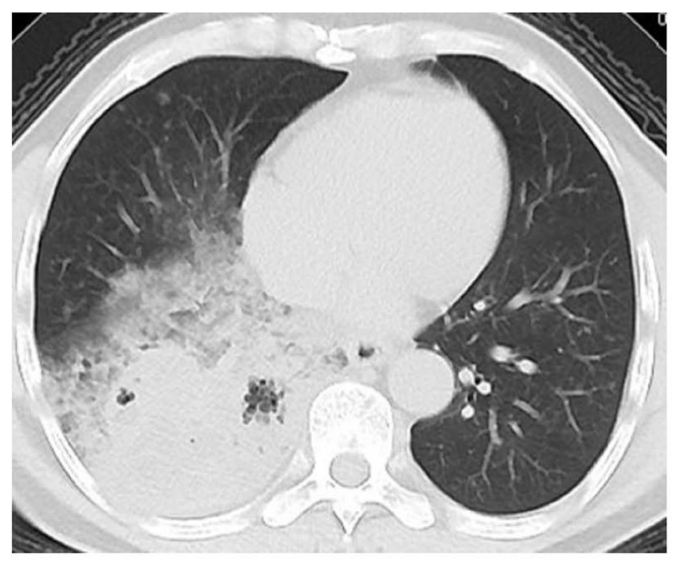

d

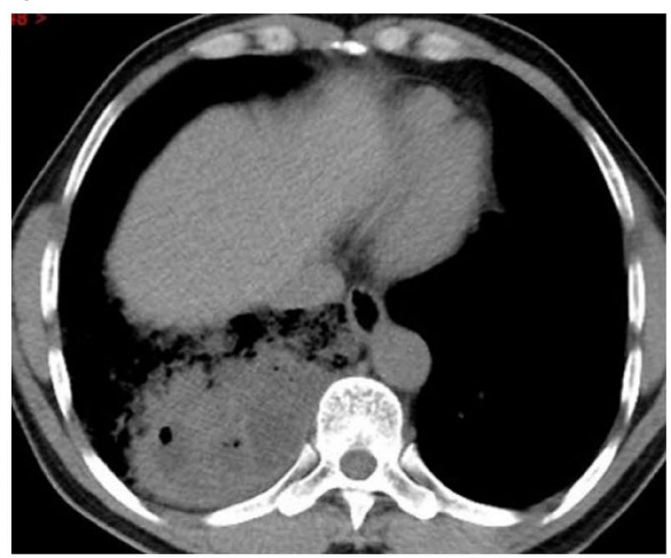

e

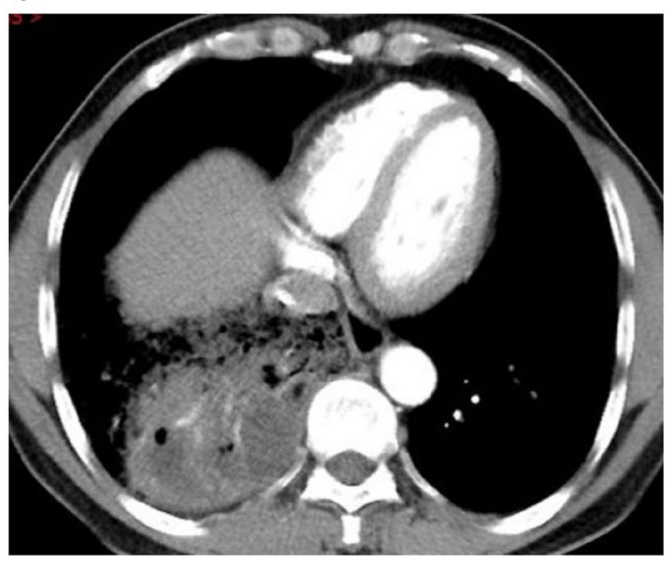

f

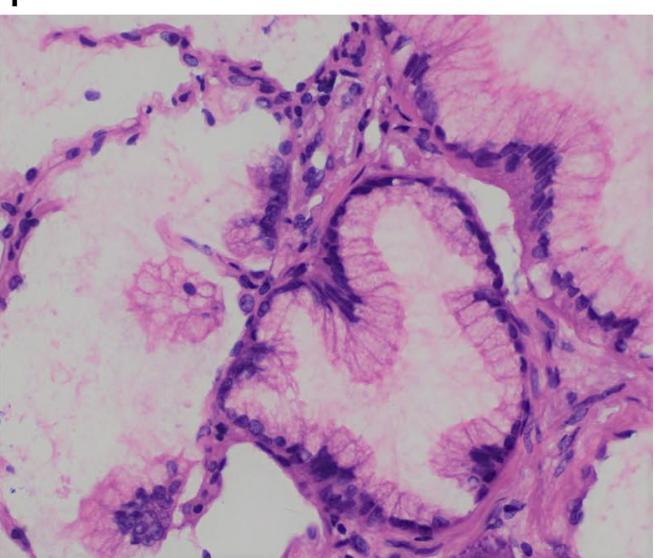

Fig. 3 D-PLADC in a 49-year-old man with cough and sputum for 6 months. a-c Axial CT images of the lung window indicate diffuse consolidation with interlobular fissure bulging, air space, GGO, irregular air bronchogram, and coexisting nodules in bilateral multiple lobes. $\mathbf{d}$-e Axial CT images of the mediastinal window at unenhanced scan (d) and arterial phase (e) indicate hypodense sign and CT angiogram within consolidation, respectively. $\mathbf{f}$ Photomicrograph (hematoxylin and eosin staining, $\times 400$ ) of biopsy specimens confirmed invasive mucinous adenocarcinoma

L-PLADC can be explained by the following: the majority of patients were female and non-smokers; most patients were with adenocarcinoma and had acinar, papillary, and lepidic growth pattern. The presence of EGFR mutations has been clearly proved as a strong point for predicting a good response to EGFR-tyrosine kinase inhibitors 


\section{a Histological subtypes of patients with b Histological subtypes of patients with L-PLADC $(n=103)$ D-PLADC $(n=26)$}

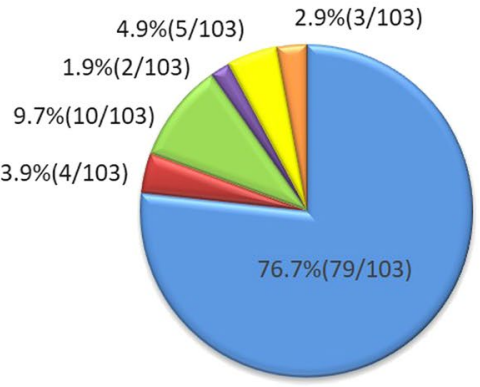

$\square$ acinar-predominant subtype

$\square$ papillary-predominant subtype

$\square$ lepidic-predominant subtype

\author{
\solid-predominant subtype \\ micropapillary-predominant subtype \\ $\square$ mucinous adenocarcinoma
}

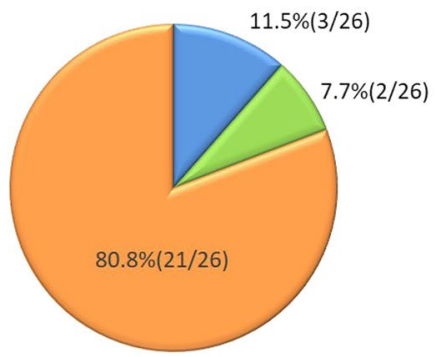

$\square$ acinar-predominant subtype

$\square$ papillary-predominant subtype

$\square$ mucinous adenocarcinoma

Fig. 4 Distribution diagrams for histological subtypes of patients with L-PLADC and D-PLADC. Notes: Data are presented as \%

[31]. According to our results, patients with L-PLADC in advanced stages may be more likely to benefit from targeted therapy. In contrast, standard chemotherapy may still be a preferred treatment strategy for patients with D-PLADC, and targeted therapy can be given to patients with EGFR-activating mutation.

This study has several limitations that should be considered. First, the retrospective nature of this study might have resulted in selection bias; further, we only performed univariate analyses. Second, only a small number of patients with D-PLADC underwent surgical resection; thus, future studies on the histological subtypes of D-PLADC with larger sample sizes are warranted to substantiate our findings. Third, our analyses were limited to adenocarcinomas and did not address other histologic types. This was done because the majority of PLADC is found in adenocarcinomas.

\section{Conclusion}

In conclusion, our findings demonstrate that L-PLADC and D-PLADC have different clinical, imaging, and pathological characteristics. This new imaging-based classification may help improve our understanding of PLADC and develop personalized treatment plans, with concomitant implications for patient outcomes.

\footnotetext{
Abbreviations

BAC: Bronchioloalveolar carcinoma; CT: Computed tomography; D-PLADC: Diffuse pneumonic-type lung adenocarcinoma; EGFR: Epidermal growth factor receptor; GGO: Ground-glass opacity; LADC: Lung adenocarcinoma; L-PLADC: Localized pneumonic-type lung adenocarcinoma; PACS: Picture archiving and communication system; PLADC: Pneumonic-type lung adenocarcinoma.
}

\section{Authors' contributions}

1. Study concepts were carried out by QL. 2. Study design was done by QL and JH. 3. Data acquisition was done by JH, XL, JG, and XH. 4. Quality control of data and algorithms was done by TL and QL. 5. Data analysis and interpretation were carried out by $\mathrm{JH}, \mathrm{XL}, \mathrm{QL}$, and $\mathrm{XH}$. 6. Statistical analysis was done by $J H$ and $\mathrm{XH}$. 7. Manuscript editing was done by QL, JH, XL, TL, and XH. JH and $\mathrm{XH}$ contributed equally to this work and share the first authorship. All authors read and approved the final manuscript.

\section{Funding}

This study has received funding by Chongqing Science and Technology Commission (cstc2017jcyjAX0281 and cstc2016shms-ztzx10002).

\section{Availability of data and materials}

The datasets used and/or analyzed during the current study are available from the corresponding author on reasonable request.

\section{Declarations}

\section{Ethics approval and consent to participate}

The ethics committee of the First Affiliated Hospital of Chongqing Medical University approved this retrospective study, and the need for informed consent was waived owing to the retrospective nature of the study.

\section{Consent for publication}

Not applicable.

\section{Competing interests}

The authors declare that they have no competing interests.

\section{Author details}

${ }^{1}$ Department of Radiology, The First Affiliated Hospital of Chongqing Medical University, No. 1 Youyi Road, Yu zhong District, Chongqing 400016, China. ${ }^{2}$ Department of Pathology, Chongqing Medical University, No.1 Youyi Road, Yuzhong District, Chongqing 400016, China.

Received: 23 September 2021 Accepted: 25 October 2021

Published online: 17 November 2021 


\section{References}

1. Siegel RL, Miller KD, Jemal A (2020) Cancer statistics. CA Cancer J Clin 70(1):7-30

2. Pascoe HM, Knipe HC, Pascoe D et al (2018) The many faces of lung adenocarcinoma: a pictorial essay. J Med Imaging Radiat Oncol 62(5):654-661

3. Lee KS, Kim Y, Han J et al (1997) Bronchioloalveolar carcinoma: clinical, histopathologic, and radiologic findings. Radiographics 17(6):1345-1357

4. Jung JI, Kim H, Park SH et al (2001) CT differentiation of pneumonic-type bronchioloalveolar cell carcinoma and infectious pneumonia. $\mathrm{Br} J$ Radiol 74(882):490-494

5. Aquino SL, Chiles C, Halford P (1998) Distinction of consolidative bronchioloalveolar carcinoma from pneumonia: do CT criteria work? AJR Am J Roentgenol 171(2):359-363

6. Im JG, Han MC, Yu EJ et al (1990) Lobar bronchioloalveolar carcinoma: "angiogram sign" on CT scans. Radiology 176(3):749-753

7. Kim TH, Kim SJ, Ryu YH et al (2006) Differential CT features of infectious pneumonia versus bronchioloalveolar carcinoma (BAC) mimicking pneumonia. Eur Radiol 16(8):1763-1768

8. Liu J, Shen J, Yang C et al (2015) High incidence of EGFR mutations in pneumonic-type non-small cell lung cancer. Medicine (Baltimore) 94(8):e540

9. Goldstraw P, Chansky K, Crowley J et al (2016) The IASLC lung cancer staging project: proposals for revision of the TNM stage groupings in the forthcoming (eighth) edition of the TNM classification for lung cancer. J Thorac Oncol 11(1):39-51

10. Travis WD, Brambilla E, Noguchi M et al (2011) International association for the study of lung cancer/American Thoracic Society/European respiratory society international multidisciplinary classification of lung adenocarcinoma. J Thorac Oncol 6(2):244-285

11. Gazdar AF, Minna JD (1997) Cigarettes, sex, and lung adenocarcinoma. J Natl Cancer Inst 89(21):1563-1565

12. Sakurai H, Asamura H, Miyaoka E et al (2014) Japanese Joint Committee of Lung Cancer Registry. Differences in the prognosis of resected lung adenocarcinoma according to the histological subtype: a retrospective analysis of Japanese lung cancer registry data. Eur J Cardiothorac Surg 45(1):100-107

13. Chu ZG, Sheng B, Liu MQ, Lv FJ, Li Q, Ouyang Y (2016) Differential diagnosis of solitary pulmonary inflammatory lesions and peripheral lung cancers with contrast-enhanced computed tomography. Clinics (Sao Paulo) 71(10):555-561

14. Yao Z, Zhang Y, Wu H (2019) Regulation of C-reactive protein conformation in inflammation. Inflamm Res 68(10):815-823

15. Balkwill F, Mantovani A (2001) Inflammation and cancer: back to Virchow? Lancet 357(9255):539-545

16. Grivennikov SI, Greten FR, Karin M (2010) Immunity, inflammation, and cancer. Cell 140(6):883-899

17. Oda S, Awai K, Liu D et al (2008) Ground-glass opacities on thin-section helical CT: differentiation between bronchioloalveolar carcinoma and atypical adenomatous hyperplasia. AJR Am J Roentgenol 190(5):1363-1368

18. Watanabe H, Saito H, Yokose T et al (2015) Relation between thin-section computed tomography and clinical findings of mucinous adenocarcinoma. Ann Thorac Surg 99(3):975-981
19. Tan Y, Gao J, Wu C et al (2019) CT characteristics and pathologic basis of solitary cystic lung cancer. Radiology 291(2):495-501

20. Xue X, Wang P, Xue Q et al (2012) Comparative study of solitary thinwalled cavity lung cancer with computed tomography and pathological findings. Lung Cancer 78(1):45-50

21. Zwirewich CV, Miller RR, Müller NL (1990) Multicentric adenocarcinoma of the lung: CT-pathologic correlation. Radiology 176(1):185-190

22. Akira M, Atagi S, Kawahara M, luchi K, Johkoh T (1999) High-resolution CT findings of diffuse bronchioloalveolar carcinoma in 38 patients. AJR Am J Roentgenol 173(6):1623-1629

23. Kim HY, Shim YM, Lee KS et al (2007) Persistent pulmonary nodular ground-glass opacity at thin-section CT: histopathologic comparisons. Radiology 245(1):267-275

24. Boland JM, Maleszewski JJ, Wampfler JA et al (2018) Pulmonary invasive mucinous adenocarcinoma and mixed invasive mucinous/nonmucinous adenocarcinoma-aclinicopathological and molecular genetic study with survival analysis. Hum Pathol 71:8-19. https://doi.org/10.1016/j.humpath. 2017.08.002

25. Boland JM, Wampfler JA, Yang P et al (2017) Growth pattern-based grading of pulmonary adenocarcinoma-analysis of 534 cases with comparison between observers and survival analysis. Lung Cancer 109:14-20

26. Shimizu K, Okita R, Saisho S et al (2016) Clinicopathological and immunohistochemical features of lung invasive mucinous adenocarcinoma based on computed tomography findings. Onco Targets Ther 10:153-163

27. Liu Y, Li J, Wang S et al (2019) Advanced pneumonic-type lung carcinoma: a retrospective study of clinical-radiological-pathological characteristics with survival analysis in a single Chinese hospital. Zhongguo Fei Ai Za Zhi 22(6):329-335

28. Liu Y, Kim J, Balagurunathan Y et al (2016) Radiomic features are associated with EGFR mutation status in lung adenocarcinomas. Clin Lung Cancer 17(5):441-448.e6

29. Lee HJ, Kim YT, Kang CH et al (2013) Epidermal growth factor receptor mutation in lung adenocarcinomas: relationship with CT characteristics and histologic subtypes. Radiology 268(1):254-264

30. Yanagawa N, Shiono S, Abiko M et al (2014) The correlation of the International Association for the Study of Lung Cancer (IASLC)/American Thoracic Society (ATS)/European Respiratory Society (ERS) classification with prognosis and EGFR mutation in lung adenocarcinoma. Ann Thorac Surg 98(2):453-458

31. Rosell R, Carcereny E, Gervais R et al (2012) Erlotinib versus standard chemotherapy as first-line treatment for European patients with advanced EGFR mutation-positive nonsmall-cell lung cancer (EURTAC): a multicentre, open-label, randomised phase 3trial. Lancet Oncol 13(3):239-246

\section{Publisher's Note}

Springer Nature remains neutral with regard to jurisdictional claims in published maps and institutional affiliations.

\section{Submit your manuscript to a SpringerOpen ${ }^{\circ}$ journal and benefit from:}

- Convenient online submission

- Rigorous peer review

- Open access: articles freely available online

- High visibility within the field

Retaining the copyright to your article

Submit your next manuscript at springeropen.com 